\title{
Metodología para la estimación del potencial DE BIOMASA EN CIENFUEGOS CON FINES ENERGÉTICOS
}

\author{
Methodology for the estimation of biomass potential \\ in Cienfuegos for EnERgy purposes \\ Reinier Jiménez Borges* \\ Eduardo Julio López Bastida** \\ Félix González Pérez**** \\ Javier Alejandro Curbelo García****
}

Recibido: 17 de julio de 2017

Aceptado: 16 de agosto de 2017

\section{Resumen}

Este artículo presenta la metodología utilizada para calcular las potencialidades presentes y futuras del total de residuos por fuente de biomasa. A partir del levantamiento de la biomasa cañera, cafetalera, arrocera y residuos aserraderos, mediante informaciones aportadas por las principales empresas productoras de la provincia de Cienfuegos, fue posible determinar la energía total entregada, así como la reducción (valorada en toneladas) de las emisiones $\mathrm{CO}_{2}$ a la atmósfera. Para el caso del bagazo, la energía total fue de $4.409 \mathrm{E}+08 \mathrm{MJ}$ y se redujo $137652.9 \mathrm{t}$ de $\mathrm{CO}_{2}$; por su parte, la energía total derivada de la cachaza fue de $7.093 \mathrm{E}+08 \mathrm{MJ}$ y se redujo 221423.9 t de $\mathrm{CO}_{2}$. Para el caso de los residuos de café y arroz y los remanentes generados por los aserraderos, estos valores fueron de 4.808E+05 MJ (150.08 t de $\left.\mathrm{CO}_{2}\right), 6.347 \mathrm{E}+07 \mathrm{MJ}\left(19813,24 \mathrm{t}\right.$ de $\left.\mathrm{CO}_{2}\right)$ y 4.108E+07 MJ (12 824.19 t de $\mathrm{CO}_{2}$ ), respectivamente.

Palabras clave: bagazo, cachaza, arroz, energía, potencialidades.

\section{Abstract}

This paper presents the methodology used to calculate the present and future potentials of the total waste by biomass source. Based on the information provided by the leading producing companies of the province of Cienfuegos, it was possible to determine the overall energy delivered, as well as the reduction (in tons) of the $\mathrm{CO}_{2}$ emissions to the atmosphere from the lifting of the biomass of sugarcane, coffee, rice, and sawdust. In the case of the bagasse, the total energy was $4.409 \mathrm{E}$ $+08 \mathrm{MJ}$ and reduced $137652.9 \mathrm{t}$ of $\mathrm{CO}_{2}$; for its part, the total energy derived from the cachaza was $7.093 \mathrm{E}+08 \mathrm{MJ}$ and decreased $221423.9 \mathrm{t}$ of $\mathrm{CO}_{2}$. For the case of waste of coffee and rice

\footnotetext{
* Ingeniero Mecánico, máster en Eficiencia Energética. Centro de Estudios de Energía y Medio Ambiente (CEEMA), Universidad de Cienfuegos. Cienfuegos, Cuba. ORCID:http://orcid.org/0000-0001-6451-8499. rjborges@ucf. edu.cu/rmeca89@gmail.com

${ }^{* *}$ Doctor en Ciencias. Profesor titular Centro de Estudios de Energía y Medio Ambiente (CEEMA), Universidad de Cienfuegos. Cienfuegos, Cuba. ORCID:http://orcid.org/0000-0003-1305-852X. kuten@ucf.edu.cu

*** Doctor en Ciencias. Profesor titular Centro de Estudios de Energía y Medio Ambiente (CEEMA), Universidad de Cienfuegos. Cienfuegos, Cuba. ORCID:http://orcid.org/0000-0003-2637-7108. fgzalez620@gmail.com

**** Ingeniero Mecánico. Unión Eléctrica Cumanayagua. ORCID:http://orcid.org/0000-0001-7859-0029
} 
and the remnants generated by sawmills, these values were $4.808 \mathrm{E}+05 \mathrm{MJ}\left(150.08 \mathrm{t}\right.$ of $\left.\mathrm{CO}_{2}\right)$, $6.347 \mathrm{E}+07 \mathrm{MJ}(19813,24 \mathrm{t}$ of CO2) and 4.108E + 07 MJ (12 824.19 t of CO2), respectively.

Keywords: bagasse, cachaza, rice, energy, potentialities

\section{INTRODUCCIÓN}

La bioenergía es en la actualidad la mayor fuente de energía renovable en la Unión Europea; casi la mitad de ese consumo total fue utilizado en el sector de la energía para la generación de electricidad y calor (Ericsson y Werner, 2016). Durante el 2015 se agregó un estimado de $147 \mathrm{GW}$ de capacidad de energía renovable -el mayor incremento anual registrado-, lo que aumentó la capacidad calorífica en alrededor de $38 \mathrm{GWt}$, así como la producción total de biocombustibles (Sawin, 2016). La utilización de estas fuentes de biomasa en la generación de electricidad es afín al desarrollo de tecnologías eficientes, lo que permite que esta producción sea competitiva con el uso de los combustibles convencionales en las condiciones específicas de los países en desarrollo (Bildirici, 2013; Brosowski et ál., 2016; Giuntoli, Caserini, Lugato, Baxter y Marelli, 2016). Por su parte, el estudio de Lewandowski, Hooijdonk, Havlickova, van Dam y Faaij (2006) evaluó el potencial de biomasa a nivel regional y estimó el potencial de energía para seis escenarios en estudio.

La literatura cuenta con numerosos estudios relacionados con la biomasa y su utilización con fines energéticos. Li, Zhou y Wang (2017) consideran el potencial de las biomasas municipales para ser aplicado como fuente de energía. El trabajo de Vavrova, Knápek, Weger, Králík y Beranovský (2017) presenta la metodología y el estudio de casos para las condiciones de la Republica Checa, en el que se usó BICOM (biomass competiveness model) ${ }^{1}$ para evaluar la competitividad económica de la utilización de la biomasa. Toklu (2017) determina el potencial de energía total de biomasa en Turquía en alrededor de 33 Mtoe y establece que la cantidad potencial de biomasa utilizable es de 17 Mtoe. La investigación de Ferreira, Monteiro, Brito y Vilarinho (2017) analiza el alcance, potencial y tecnologías relacionadas con el uso de las fuentes de biomasa; además, discute los proyectos gubernamentales y no gubernamentales relacionados con la biomasa, así como los planes y las estrategias para su promoción. Igualmente, este estudio trata sobre la situación actual de los recursos biomásicos y muestra que el potencial total estimado para varios sectores de Portugal es de 42489.7 GWh/año. Thomas, Soren, Rumjit, James y Saravanakumar (2017) examinan el potencial, objetivos y oportunidades de las biomasas en la economía india; asimismo, analizan las técnicas de conversión propia para cada una de estas fuentes, así como la factibilidad de conversión.

El trabajo de Ozcan, Öztürk, y Oguz (2017) determina la cantidad de energía eléctrica primaria obtenida del potencial de biomasa de acuerdo a diferentes tipos de fuentes de biomasas. Las biomasas evaluadas fueron los residuos sólidos municipales, cultivos energéticos, residuos de animales, entre otros. Como resultado de los métodos de cálculo, el valor total de energía primaria del biogás obtenido de las fuentes de biomasas examinadas fue de 188.21 TWh/año. El valor total de energía primaria relacionado con el potencial de las fuentes de biomasas evaluadas fue de $278.40 \mathrm{TWh} / \mathrm{año}$.

La investigación de Ozturk et ál. (2017) estima que la producción anual de desechos en Turquía es de 30 Mton. Turquía produce 1.5 Mton de biodiesel, 3 Mton de bioetanol y 2.5-4.0 billones de $\mathrm{m}^{3}$ de biogás al año. Hossen, Rahman, Kabir, Hasan y Ahmed (2017) presentan una valoración objetiva de la biomasa disponible como una fuente prominente de energía y sugieren que este potencial es una fuente de energía primaria sostenible que eventualmente puede reemplazar a largo plazo la utilización de combustibles fósiles. Se estimó que el total de biomasa disponi-

${ }^{1}$ Modelo de competitividad de la biomasa. 
ble procedente de la agricultura, desechos, industrias, animales y otras fuentes puede generar más de $3447 \mathrm{PJ}$ (>950 TWh) de energía.

El trabajo de Suzuki, Tsuji, Shirai, Hassan y Osaki (2017) analiza el potencial de energía de biomasa en Sabah, a partir de datos establecidos de la literatura, datos estadísticos y documentos disponibles. Este estudio establece que el potencial energético de la biomasa es alrededor de 267.18 GJ/año en total. Vávrová, Knápek y Weger (2017) evalúan el potencial de las biomasas para un área definida, basados en una metodología que usa detalles espaciales y datos empíricos. En este análisis se demuestra cómo este potencial depende tanto de la región, como de la composición. La investigación de Stich, Ramachandran, Hamacher y Stimming (2017) valora el potencial de generación de energía eléctrica a partir de residuos agrícolas y la ganadería, y estima el costo de la producción de electricidad a partir de estos. Los autores desarrollaron un modelo de optimización de costo para evaluar el costo-beneficio de la producción de electricidad de estas fuentes usando varias tecnologías de conversión. El estudio de Ozoegwu, Eze, Onwosi, Mgbemene y Ozor (2017), a partir de datos estadísticos del total de residuos de agricultura en Nigeria, emplea técnicas de regresión estableciendo factores de escala para el procesamiento de esos datos. Simangunsong et ál. (2017) estiman el valor económico del potencial de biomasa forestal una vez transformada en pellets en US \$5.6/ton de residuos de madera. La investigación de González-Salazar, Venturini, Poganietz, Finkenrath y Spina (2017) presenta un nuevo enfoque para la valoración del potencial de energía de la biomasa cuando la disponibilidad de datos es limitada. Loh (2016) caracteriza las numerosas maneras en las que la biomasa, a partir del aceite de palma en Malasia, puede ser utilizado como combustible; igualmente, evalúa los datos recolectados en función del potencial energético, las tecnologías de conversión relacionada con este tipo de biomasa y los posibles problemas relacionados con la combustión. Al respecto de este estudio, hay que considerar que la industria de aceite de palma en este país es una de las más grandes generadoras de biomasa lignocelulósica (más del $90 \%$ del total de biomasa en el país se deriva de 5.4 millones de hectáreas).

Otros estudios, como el de Giuntoli et ál. (2016), utilizan la evaluación del análisis del ciclo de vida atribucional (A-LCA, sigla en inglés) para analizar el potencial de mitigación del cambio climático de tres plantas que utilizan biomasa residual, comparado con otras que utilizan combustibles fósiles en la Unión Europea. Phillips et ál. (2016) estudian la viabilidad de la biomasa como combustible en el Reino Unido, en el que estimaron un total de $500 \mathrm{kt} / \mathrm{año}$. La investigación de Román-Figueroa, Montenegro y Paneque (2016) estima la cantidad de energía eléctrica que puede producirse a partir de la paja de trigo en la región Araucana de Chile. En este estudio se determinó que el total de biomasa disponible fue de 0.622 millones de toneladas (el distrito de Quilquén el de mayor producción con 0.27 millones de toneladas). Nakomcic-Smaragdakis, Cepic y Dragutinovic (2016) estiman el potencial de biomasa sólida (residuos de cosecha) de la provincia autónoma de Vojvodina (República de Serbia). El potencial fue evaluado en 6.45 Mton/año, en el que solo 2.45 Mton/año puede ser utilizado con fines energéticos, es decir, el $37.98 \%$.

E1 sector de la biomasa desempeña un rol estratégico en las directivas de energías renovables, que es acorde con los planes de acción de energía renovable nacionales elaborados en conjunto con la directiva 2009/28/EC ${ }^{2}$. Paiano y Lagioia (2016) evalúan la cantidad de biomasa residual disponible (particularmente, lignocelulósica) en el territorio italiano. Esta investigación estableció que el total de energía final que puede ser generada de 22208.46 t/año de biomasa residual estimada es cerca del $2.7 \%$ del consumo energético bruto para el 2013, lo que significa un ahorro de emisiones de gases de efecto invernadero proveniente de la generación con biomasa de $52 \mathrm{Mt} \mathrm{CO}_{2}$ eq/año para el territorio italiano en general.

${ }^{2}$ Directiva del Consejo Europeo 2009/28/EC 
En Cuba la principal fuente de energía renovable (además de la energía solar) es la biomasa, ya que no existen grandes ríos, ni zonas con altas velocidades del viento. Si bien el mayor potencial energético lo tiene la biomasa cañera, existen otras fuentes que tienen importancia en el orden local o que su aprovechamiento resulta conveniente desde el punto de vista medioambiental, ya que en el país constituye una prioridad el fomento y utilización de las fuentes renovables de energía. Actualmente, según Guerra (2016), es baja la utilización de las fuentes renovables de energía; estas fuentes solo se producen el $4.3 \%$ de la electricidad del país (la biomasa genera el $3.5 \%$ ).

La provincia de Cienfuegos está localizada en la zona central de Cuba y ocupa un área de 4178 $\mathrm{km}^{2}$ (417 $800 \mathrm{ha}$ ). Cienfuegos tiene una economía variada que está basada fundamentalmente en la agricultura, la industria y el turismo (Sagastume et ál., 2016). La provincia cuenta con una estadística generalizada de la situación de las biomasas, de ahí que es de gran importancia contar en detalle con información aportada de un número de empresas productoras del territorio que se utilice como base para la estimación del potencial energético de cada una de estas biomasas. El objetivo fundamental de esta investigación es estimar la cantidad de biomasa disponible en la provincia que puede ser utilizada con fines energéticos.

\section{Metodología}

La biomasa está asociada al fomento de energías procedente de fuentes renovables, como: la fracción biodegradable de los productos, desechos y residuos de origen biológico procedentes de actividades agrarias (incluidas las sustancias de origen vegetal y animal), la silvicultura y la industrias (incluidas la pesca y la acuicultura), así como la fracción biodegradable de los residuos industriales y urbanos (Pinedo, 2013). Este trabajo abarca algunas de estas fuentes de biomasa; sin embargo, el estudio no considera fuentes como los residuos sólidos urbanos o los residuos procedentes de animales. Para este caso, las fuentes seleccionadas en el estudio fueron: residuos agrícolas de la cosecha (cáscara de arroz, paja de caña, cascarilla de café), bioproductos de la industria azucarera (bagazo, cachaza) y residuos forestales (residuos de aserraderos).

Para determinar la energía total por cada fuente de biomasa se utiliza la expresión 1.

$$
E_{\text {Total }}=B_{\text {prod }}+P C S_{b . s}
$$

Donde $\mathrm{E}_{\text {Total }}$ es energía total (MJ); $\mathrm{B}_{\text {prod }}$, cantidad de biomasa producida $(\mathrm{kg})$, y $\mathrm{PCS}_{\mathrm{b} . s}$, poder calórico superior en base seca $(\mathrm{MJ} / \mathrm{kg})$

La energía útil, por otra parte, puede ser determinada mediante la ecuación 2.

$$
E_{\text {Utili }}=B_{\text {comb }}^{*} P C S_{b . s}
$$

Donde $\mathrm{E}_{\text {Útil }}$ es energía útil (MJ) y $\mathrm{B}_{\text {prod }}$ es biomasa utilizada como combustible (kg).

Por tanto, la eficiencia con que se utiliza la energía puede ser determinada mediante la ecuación 3.

$$
\eta=\frac{E_{\text {Util }}}{E_{\text {Total }}}
$$

Con el aprovechamiento de las fuentes de biomasa desde el punto de vista energético se logra una reducción en el empleo de combustibles fósiles para un determinado proceso; esto permite la reducción de las emisiones de $\mathrm{CO}_{2}$ a la atmosfera y, por consiguiente, la mitigación del cambio 
climático. La cantidad de $\mathrm{CO}_{2}$ que se deja de emitir a la atmosfera puede ser determinada mediante la ecuación 4 (Salazar, Mockey y Canal, 2009):

$$
E=E_{a}^{*} F E
$$

Dónde $\mathrm{E}$ es emisiones ( $\mathrm{kWh} / \mathrm{año}) ; \mathrm{FE}$, factor de emisión (0.001127 tCO2/kWh), y E_a, energía ahorrada ( $\mathrm{kWh} / \mathrm{año})$

\section{Estimación de los bioproductos de la industria azucarera}

Desde el punto de vista estratégico, la estimación de la biomasa potencial puede ser una herramienta eficaz para conocer la cantidad de residuos y realizar una planificación más acertada. Considerando las cifras de producción de la caña y los residuos generados, así como con el área total de caña sembrada y los rendimientos, se puede conocer el potencial con el que se cuenta. La tabla 1 presenta la producción de caña para las últimas tres zafras.

Tabla 1. Producción de caña de azúcar en toneladas ( $t$ ) en las últimas tres zafras

\begin{tabular}{cc}
\hline Zafras & Producción de caña (t) \\
\hline $2013-2014$ & 1529771.51 \\
$2014-2015$ & 1642767.82 \\
$2015-2016$ & 1630540.78 \\
\hline Total & 4803080.11 \\
\hline
\end{tabular}

Fuente: Oficina Estadística de AZCUBA (2016).

Como se observa en la tabla 1, la tendencia ha sido el incremento en la producción de caña. El incremento entre la zafra 2013-2014 y la zafra 2015-2016 fue de un 6.2 \%, aproximadamente. En la tabla 2 se muestra la tenencia y uso de la tierra para el cultivo de la caña para poder determinar los rendimientos de caña por área de tierra sembrada, así como las áreas destinadas al cultivo. Se cuenta con 65269.43 ha de tierras destinadas a la siembra de caña de azúcar, de las que solo 45303.44 ha son cultivadas

Tabla 2. Tenencia y uso de la tierra para el cultivo en hectáreas (ha) de la caña de azúcar

\begin{tabular}{lr}
\hline \multicolumn{1}{c}{ Indicadores } & Total (ha) \\
\hline Caña de azúcar & 65269.43 \\
Superficie cultivada & 45303.44 \\
Bancos de semillas & 99.00 \\
En rotación & 224.41 \\
Guardarrayas & 4627.92 \\
Vacías & 6733.59 \\
Ociosas & 8281.07 \\
De ellas: con marabú & 7624.21 \\
\hline
\end{tabular}

Fuente: Centro Nacional de Control de Tierras (2015). 
En función de los valores de producción de caña molida y el área destinada para el cultivo se obtuvieron los rendimientos promedios de caña por hectárea (ver tabla 3), con los que se buscan alternativas para obtener un mayor rendimiento en las cosechas y lograr como meta mínima 35 t de caña/ha en cada unidad productora.

Tabla 3. Rendimiento de la caña de azúcar en los años de estudio

\begin{tabular}{cccc}
\hline Zafras & Producción de caña(t) & Área Cultivada(ha) & Rendimiento (t/ha) \\
\hline $2013-2014$ & 1529771.51 & 45303.44 & 33.77 \\
$2014-2015$ & 1642767.82 & 45303.44 & 36.26 \\
$2015-2016$ & 1630540.78 & 45303.44 & 35.99 \\
\hline
\end{tabular}

Los bioproductos estimados de la industria azucarera fueron el bagazo y la cachaza. En la provincia de Cienfuegos se contó con 1571600 t de bagazo, de las que solo se utilizaron 1506447 t, de manera que el promedio fue de $523866 \mathrm{t}$ de bagazo producido por año (tabla 4).

Tabla 4. Bagazo producido y utilizado como combustible en toneladas en las tres últimas zafras

\begin{tabular}{cccc}
\hline Zafras & Bagazo producido $(t)$ & Bagazo combustible $(t)$ & Bagazo sobrante $(t)$ \\
\hline $2013-2014$ & 508347 & 487303 & 21044 \\
$2014-2015$ & 449356 & 430735 & 18621 \\
$2015-2016$ & 613897 & 588409 & 25488 \\
\hline Total & 1571600 & 1506447 & 65153 \\
\hline
\end{tabular}

Fuente: Oficina Estadística de AZCUBA (2016).

Por otra parte, la tabla anterior muestra que el bagazo sobrante es de $65153 \mathrm{t}$; remanente que puede ser empleado como materia prima en la industria de derivados. El carácter renovable del bagazo ha estimulado en las últimas décadas un proceso acelerado de desarrollo para su utilización en las producciones de pulpa papel y productos aglomerados.

El aprovechamiento actual que tiene el bagazo es para la producción de energía térmica mediante el proceso de combustión (debido al valor calórico de este proceso). La tabla 5 presenta las 144092.4 t de cachaza que ha generado la empresa azucarera en las últimas tres zafras.

Tabla 5. Comportamiento de la cachaza en las tres últimas zafras.

\begin{tabular}{cc}
\hline Zafras & Cachaza (t) \\
\hline $2013-2014$ & 45893.14 \\
$2014-2015$ & 49283.03 \\
$2015-2016$ & 48916.22 \\
\hline Total & 144092.4 \\
\hline
\end{tabular}

Fuente: Oficina Estadística de AZCUBA (2016). 


\section{Estimación de los residuos agrícolas de la cosecha (RAC)}

Los residuos agrícolas estimados (RAC) en el estudio fueron la cascarilla de café y la cáscara de arroz, además de la paja de caña, que por sus ventajas como recurso renovable y su posible empleo para la producción de energía es otro residuo a considerar. La tabla 6 presenta las estadísticas sobre la cantidad de paja de caña en los centros de limpieza donde se procesa y limpia la caña, que generan entre 6 y $8 \%$ de los residuos, los cuales en su gran mayoría se queman y en menor medida se emplean como alimento animal.

Tabla 6. Paja en los centros de limpieza en las tres últimas zafras

\begin{tabular}{cc}
\hline Cosechas & Pajas en centros de limpieza(t) \\
\hline $2013-2014$ & 80264.40 \\
$2014-2015$ & 82849.70 \\
$2015-2016$ & 87345.75 \\
\hline Total & 250460.86 \\
\hline
\end{tabular}

Fuente: Oficina Estadística de AZCUBA (2016).

Además de la paja de caña, los cultivos de café son otra de las fuentes de biomasa. En la tabla 7 se muestra la composición del café mediante el proceso de beneficio húmedo en los últimas tres cosechas.

Tabla 7. Beneficio húmedo del café en las últimas tres cosechas

\begin{tabular}{ccccc}
\hline Cosechas & Unidad & Cereza & Cáscara & Mucílago \\
\hline $2013-2014$ & $\mathrm{t}$ & 132.16 & 54.98 & 20.62 \\
$2014-2015$ & $\mathrm{t}$ & 104.68 & 43.55 & 16.33 \\
$2015-2016$ & $\mathrm{t}$ & 121.87 & 50.70 & 19.01 \\
\hline Total & & 358.71 & 149.22 & 55.96 \\
\hline
\end{tabular}

Fuente: Oficina Estadística de la Empresa Procesadora de Café (2016).

La tabla 8 evidencia que en las últimas tres cosechas se ha producido 334.62 t de café y que se han generado algo más de $94.38 \mathrm{t}$ de cascarilla.

Tabla 8. Producción de café y la generación de cascarilla en las últimas tres cosechas

\begin{tabular}{cccc}
\hline Cosecha & Pergamino seco $(t)$ & Café oro $(t)$ & Cascarilla $(t)$ \\
\hline $2013-2014$ & 178.21 & 139.00 & 39.21 \\
$2014-2015$ & 133.92 & 104.46 & 29.46 \\
$2015-2016$ & 116.88 & 91.17 & 25.71 \\
\hline Total & 429.01 & 334.62 & 94.38 \\
\hline
\end{tabular}

Fuente: Oficina Estadística de la Empresa Procesadora de Café (2016) 
Según el Centro Nacional de Control de la Tierra (2015), en Cienfuegos existen 7440.28 ha destinadas al sembrado de arroz, que generaron una producción para el año 2016 de aproximadamente 10515.70 t (Oficina Estadística Empresa de Granos, 2017). Según los datos consultados, la zona de Aguada de Pasajeros fue la de mayor producción. La tabla 9 presenta los valores de las producciones de arroz y de los desechos producidos necesarios para estimar el potencial energético de estas fuentes.

Tabla 9. Producción en toneladas de arroz y subproductos en la provincia de Cienfuegos en el 2016

\begin{tabular}{|c|c|c|c|c|c|c|}
\hline \multirow{2}{*}{\begin{tabular}{l}
\multicolumn{1}{c}{ Entrada } \\
Arroz (cáscara) \\
(t) \\
\end{tabular}} & \multirow{2}{*}{$\begin{array}{l}\text { Obtenido } \\
\text { Arroz (listo) } \\
\text { (t) }\end{array}$} & \multirow{2}{*}{$\begin{array}{l}\text { Rend. Industrial } \\
\qquad(\%)\end{array}$} & \multicolumn{3}{|c|}{ Residuos } & Desechos \\
\hline & & & Polvo(t) & Cabecilla (t) & Cuarto grano(t) & Cascarilla (t) \\
\hline 16019.94 & 10515.70 & 65.6 & 1349.78 & 255.57 & 52.364 & 3846.52 \\
\hline
\end{tabular}

Fuente: Oficina Estadística Empresa de Granos (2016)

Uno de los usos de la cascarilla de arroz es la producción de calor mediante la combustión, debido al contenido energético que esta tiene (15,2-16,5 MJ/kg) (Gutiérrez et ál., 2016).

\section{Estimación de los residuos agroforestales}

Para la determinación de los residuos agroforestales se evalúo el total de madera aserrada en los últimos tres años. La tabla 10 muestra el comportamiento de la madera aserrada por la empresa procesadora.

Tabla 10. Madera aserrada en los tres últimos años

\begin{tabular}{lccccc}
\hline & U/M & $\mathbf{2 0 1 4}$ & $\mathbf{2 0 1 5}$ & $\mathbf{2 0 1 6}$ & Rend. \% \\
\hline Total & $\mathrm{m}^{3}$ & 2663.90 & 2783.70 & 2625.90 & 55 \\
Aserríos de tecnología atrasada & $\mathrm{m}^{3}$ & 1065.56 & 1113.48 & 1050.36 & 53 \\
\hline Aserríos de tecnología avanzada & $\mathrm{m}^{3}$ & 1598.34 & 1670.22 & 1575.54 & 55 \\
\hline
\end{tabular}

Fuente: Oficina Estadística de la Empresa Forestal Integral Cienfuegos (2016).

En este trabajo fueron analizados los residuos en el proceso de aserrío de la madera. En la tabla 11 se indican las cantidades de serrín recolectadas y los trozos de madera y costaneras que se obtuvieron, que en total alcanzaron residuos en los tres años de $6827.162 \mathrm{~m}^{3}$. Según Bucheli, Ordóñez y Muñoz (2016), la densidad aparente (que depende de la compactación) para los residuos aserraderos es de $160 \mathrm{~kg} / \mathrm{m}^{3}$ (coníferas), con la que se obtiene una masa total de residuos de $1092320 \mathrm{~kg}$.

Tabla 11. Cantidades totales de residuos en los últimos cuatro años en Cienfuegos

\begin{tabular}{lcccc}
\hline \multicolumn{5}{c}{$\mathbf{2 0 1 4}$} \\
\hline & Madera bolo & Total residuos & Serrín & Costanera \\
\hline Total & 4916.56 & 2252.66 & 1943.11 & 309.55 \\
Aserríos de tecnología atrasada & 2010.49 & 944.93 & 831.53 & 113.39 \\
Aserríos de tecnología avanzada & 2906.07 & 1307.73 & 1111.57 & 196.15 \\
\hline & Madera bolo & Total residuos & Serrín & Costanera \\
\hline & 5137.66 & 2353.96 & 2030.49 & 323.47 \\
\hline Total & 2100.9 & 987.42 & 868.93 & 118.49 \\
Aserríos de tecnología atrasada & 3036.76 & 1366.54 & 1161.56 & 204.98 \\
\hline
\end{tabular}




\begin{tabular}{lcccc}
\hline \multicolumn{5}{c}{2016} \\
\hline & Madera Bolo & Total residuos & Aserrín & Costanera \\
\hline Total & 4846.42 & 2220.52 & 1915.39 & 305.13 \\
Aserríos de tecnología atrasada & 1981.81 & 931.45 & 819.67 & 111.77 \\
Aserríos de tecnología avanzada & 2864.61 & 1289.07 & 1095.71 & 193.36
\end{tabular}

Fuente: Oficina Estadística de la Empresa Forestal Integral Cienfuegos (2016).

\section{Resultados}

Para la estimación de la cantidad de energía total entregada por fuente de biomasa se deben de conocer las composiciones químicas y los poderes calóricos, es decir, cuanta energía es capaz de entregar por unidad de masa. La tabla 12 presenta la composición química (por ciento de cenizas) y los poderes calóricos (superior e inferior) en base seca.

Tabla 12. Composición química y poderes calóricos para estas biomasas en Cuba (base seca)

\begin{tabular}{|c|c|c|c|c|c|c|c|}
\hline Biomasa & HR(\%) & $\mathrm{C}(\%)$ & $H(\%)$ & $O(\%)$ & $\begin{array}{c}\text { Cenizas } \\
(\%)\end{array}$ & $\begin{array}{l}\text { PClb.s } \\
\text { (MJ/kg) }\end{array}$ & $\begin{array}{l}\text { PCSb.s } \\
(\mathrm{MJ} / \mathrm{kg})\end{array}$ \\
\hline Bagazo & 50 & 47.2 & 7.0 & 43.1 & 2.7 & 15.8 & 17.3 \\
\hline Cachaza & $70-80$ & 32.5 & 2.2 & 2.2 & 14.5 & 8.8 & 14.5 \\
\hline Paja de caña & 45 & 43.5 & 6.1 & 41.1 & 9.3 & 15.7 & 17.2 \\
\hline Cascarilla de Café & 7.6 & 50.3 & 5,3 & 43.8 & 0.5 & 17.5 & 18.7 \\
\hline Cáscara de arroz & $8-10$ & 38.2 & 5.6 & 33.7 & 22.5 & 15.2 & 16.5 \\
\hline $\begin{array}{l}\text { Residuos } \\
\text { aserraderos }\end{array}$ & 15 & 51.4 & 5.7 & 38.7 & 3.8 & 19.2 & 18.5 \\
\hline
\end{tabular}

Fuente: Gutiérrez et ál. (2016).

A partir de las cantidades de biomasas estimadas y la composición química y los valores calóricos de estas biomasas generadas en Cuba (ver tabla 12), se puede determinar la energía total de cada fuente mediante la expresión 1, así como la energía útil puede ser hallada mediante la expresión 2 (ver expresiones en el apartado "Metodología"). De las fuentes de biomasas presentadas, solo el bagazo de caña es utilizado como combustible en los generadores de vapor de los centrales azucareros; en este caso, la $\mathrm{E}_{\text {util }}$ fue de 8,69 x109 MJ para una eficiencia de utilización $\eta$ de un $95 \%$. La tabla 13 presenta los resultados de la cantidad de energía entregada para cada fuente de biomasa correspondiente al año 2016.

Tabla 13. Cantidad total de energía entregada por fuente de biomasa.

\begin{tabular}{llc}
\hline \multicolumn{1}{c}{ Fuente de biomasa } & UM & Etotal \\
\hline Paja de caña & $\mathrm{MJ}$ & $1.508 \mathrm{E}+09$ \\
Cachaza & $\mathrm{MJ}$ & $7.093 \mathrm{E}+08$ \\
Bagazo & $\mathrm{MJ}$ & $4.409 \mathrm{E}+08$ \\
Cáscara de arroz & $\mathrm{MJ}$ & $6.347 \mathrm{E}+07$ \\
Residuos aserraderos & $\mathrm{MJ}$ & $4.108 \mathrm{E}+07$ \\
Cascarilla de café & $\mathrm{MJ}$ & $4.808 \mathrm{E}+05$ \\
\hline
\end{tabular}


Generalmente, la paja de caña presenta la mayor cantidad de energía total entregada 1.508 $\mathrm{E}+09 \mathrm{MJ}$, que es un $47 \%$ superior a la cachaza. Es importante destacar que la industria azucarera representa la principal fuente generadora de residuos (principalmente con la paja de caña en el territorio) y es la de mayor posibilidad de entrega de energía con un total de aproximadamente 2,69E+09 MJ. E1 bagazo sobrante no es aprovechado con fines energéticos actualmente; sin embargo, es necesario considerar su combustión para la producción de calor y electricidad (cogeneración), ya que puede ser aprovechados 4.409E+08 MJ.

Para el cálculo de la reducción de las emisiones de $\mathrm{CO}_{2}$ solo se evalúa el impacto en las emisiones de la generación de electricidad en Cuba (GEC), cuyo factor de emisión es de 0.001127 t de $\mathrm{CO}_{2} / \mathrm{kWh}$. Dado que las emisiones de biomasa $\mathrm{CO}_{2}$ son consideradas neutras, es decir, las emisiones de combustión de biomasa equivalen a la masa de $\mathrm{CO}_{2}$ absorbida durante su cultivo (Sagastume et ál., 2016).

La cantidad de $\mathrm{tCO}_{2}$ dejados de emitir a la atmósfera determinada por la expresión 4 se presenta en la tabla 14.

Tabla 14. Cantidad total de $\mathrm{CO}_{2}$ dejados de emitir por fuente de biomasa

\begin{tabular}{llr}
\hline \multicolumn{1}{c}{ Fuente de Biomasa } & UM & Cantidad \\
\hline Paja de caña & $\mathrm{tCO}_{2}$ & 470611 \\
Cachaza & $\mathrm{tCO}_{2}$ & 221424 \\
Bagazo & $\mathrm{tCO}_{2}$ & 137653 \\
Cáscara de arroz & $\mathrm{tCO}_{2}$ & 19813 \\
Residuos aserraderos & $\mathrm{tCO}_{2}$ & 12824 \\
Cascarilla de café & $\mathrm{tCO}_{2}$ & 150 \\
\hline
\end{tabular}

El residuo que mayor cantidad de $\mathrm{CO}_{2}$ dejó de emitir a la atmósfera fue la paja de caña con $470611 \mathrm{tCO}_{2}$ eq. Para el caso de los residuos de industria azucarera, en conjunto dejaron de emitir $829688 \mathrm{tCO}_{2}$ eq, lo que evidencia la importancia de la utilización de la biomasa con fines energéticos como una alternativa a la sustitución de los combustibles fósiles para la generación de energía.

\section{Conclusiones}

Esta investigación propuso como metodología para la valoración del potencial de biomasa con fines energéticos en Cienfuegos: estimar la cantidad de biomasa disponible para calcular la cantidad de energía total que puede ser generada y la reducción (valorada en toneladas) de las emisiones $\mathrm{CO}_{2}$ a la atmósfera.

La producción de bagazo derivado de la caña de azúcar en el último año fue de $25488 \mathrm{t}$, lo que equivale a 4.409E+08 MJ y a una reducción de 137652.9 t de $\mathrm{CO}_{2}$. La producción de cachaza obtenida fue de $48916,22 \mathrm{t}$, lo que equivale a 7.093E+08 MJ y a una reducción de 221423.9 t de $\mathrm{CO}_{2}$. En los centros de limpieza de la provincia se procesaron un total de 87345,75 t de paja, lo que generó $1.508 \mathrm{E}+09 \mathrm{MJ}$ y la reducción de 470611 t de $\mathrm{CO}_{2}$.

Uno de los principales residuos que se utiliza con fines energéticos es la cascarilla de café, que fue de 25.71 t, lo generó $4.808 \mathrm{E}+05 \mathrm{MJ}$ y la reducción de 150.08 t de $\mathrm{CO}_{2}$. El total de cascarilla de arroz fue de $3846,52 \mathrm{t}$, lo que equivale a 6,347E+07 MJ y a una reducción de 19 813,24 t de $\mathrm{CO}_{2}$. $\mathrm{E} 1$ total de residuos aserraderos para el último año fue de $2220.52 \mathrm{t}$, lo que generó $4.108 \mathrm{E}+07 \mathrm{MJ}$ y la reducción de12 824.19 t de $\mathrm{CO}_{2}$. 


\section{RefERENCIAS}

Baxter, D.,y Marelli,L.,(2016). Climate change impacts of power generation from residual biomass. Biomass and Bioenergy, 89, 146-158. doi: http://dx.doi.org/10.1016/j.biombioe.2016.02.024

Bildirici, M. (2013). Economic growth and biomass energy. Biomass and Bioenergy, 50, 19-24. doi: http://dx.doi.org/10.1016/j.biombioe.2012.09.055

Brosowski, A., Mantau, U., Mahro, B., Erdmann, G., Adler, P., Stinner, W., Reinhold, G., Hering, T., y Blanke, C. (2016). A review of biomass potential and current utilisation-Status quo for 93 biogenic wastes and residues in Germany. Biomass and Bioenergy, 95, 1-16. doi: http:// dx.doi.org/10.1016/j.biombioe.2016.10.017

Bucheli, A. D. P., Ordóñez, J. C. G., \& Muñoz, J. F. C. (2016). Metodología para el aprovechamiento del potencial energético con biomasa forestal en el departamento de Nariño: Caso de Estudio. Ingeniería Solidaria, 12(20). doi: http://dx.doi.org/10.16925/in.v19i20.1415

Ericsson. K. y Werner, S. (2016). The introduction and expansion of biomass use in Swedish district heating systems. Biomass and Bioenergy, 94, 57-65. doi: http://dx.doi.org/10.1016/j. biombioe.2016.08.011

Ferreira, S., Monteiro, E., Brito, P., y Vilarinho, C. (2017). Biomass resources in Portugal: current status and prospects. Renewable and Sustainable Energy Reviews, 78, 1221-1235. doi: http:// dx.doi.org/10.1016/j.rser.2017.03.140Giuntoli, J., Caserini, A., Lugato,

Gonzalez-Salazar, M., Venturini, M., Poganietz, W., Finkenrath, M., y Spina, P. (2016). Methodology for improving the reliability of biomass energy potential estimation. Biomass and Bioenergy, 88. doi: http://dx.doi.org/10.1016/j.biombioe.2016.03.026

Guerra, R. (2016). Cuba. Cartelera de oportunidades de inversión extranjera 2016-2017. Cuba: ProCuba y Cámara de Comercio de la república de Cuba.

Hossen, M., Rahman, A., Kabir, A., Hasan, M.., y Ahmed, S. ( (2017). Systematic assessment of the availability and utilization potential of biomass in Bangladesh. Renewable and Sustainable Energy Reviews, 67, 94-105. doi: http://dx.doi.org/10.1016/j.rser.2016.09.008

Lewandowski, I., van Hooijdonk, A., Havlickova, K., van Dam, J., y Faaij, A. (2006). The potential biomass for energy production in the Czech Republic. Biomass and Bioenergy, 30, 405-421. doi: 10.1016/j.biombioe.2005.11.020

Li, Y., Zhou, L. W., y Wang, R. (2017). Urban biomass and methods of estimating municipal biomass resources. Renewable and Sustainable Energy Reviews, 80, 1017-1030. doi: http:// dx.doi.org/10.1016/j.rser.2017.05.214

Loh, S. K. (2016). The potential of the Malaysian oil palm biomass as a renewable energy source. Energy Conversion and Management. 1-14. doi: http://dx.doi.org/10.1016/j.enconman.2016.08.081 (artículo en revisión)

Nakomcic-Smaragdakis, B., Cepic, Z., y Dragutinovic, N. (2016). Analysis of solid biomass energy potential in Autonomous Province of Vojvodina. Renewable and Sustainable Energy Reviews, 57, 186-191. doi: http://dx.doi.org/10.1016/j.rser.2015.12.118

Oficina Estadística de AZCUBA. (2016). Análisis multivariado de los residuos de la caña de azúcar. Cuba: AZCUBA. 
REINIER JIMÉNEZ, EDUARDO JULIO LÓPEZ, FÉLIX GONZÁLEZ, JAVIER ALEJANDRO CURBELO

Oficina Estadística de la Empresa Forestal Integral Cienfuegos. (2016). Madera aserrada y residuos generados en los tres últimos años. Cuba: Oficina Estadística de la Empresa Forestal Integral Cienfuegos

Oficina Estadística de la Empresa Procesadora de Café. (2016) Producciones de café y sus residuales anuales. Cuba: Oficina Estadística de la Empresa Procesadora de Café

Oficina Estadística Empresa de Granos. (2016). Cierre anual de las producciones de arroz y sus residuales. Cienfuegos, Cuba: Oficina Estadística Empresa de Granos

Ozcan, M., Öztürk, S., y Oguz, Y. (2017). Potential evaluation of biomass-based energy sources for Turkey. Engineering Science and Technology, an International Journal, 18(2), 178-184. doi: http://dx.doi.org/10.1016/j.jestch.2014.10.003

Ozoegwu, C., Eze, C., Onwosi, C., Mgbemene, C., y Ozor, P. (2017). Biomass and bioenergy potential of cassava waste in Nigeria: Estimations based partly on rural-level garri processing case studies. Renewable and Sustainable Energy Reviews, 72, 625-638. doi: http://dx.doi. org/10.1016/j.rser.2017.01.031

Paiano, A., y Lagioia, G. (2016). Energy potential from residual biomass towards meeting the EU renewable energy and climate targets. The Italian case. Energy Policy, 91, 161-173. doi: http://dx.doi.org/10.1016/j.enpol.2015.12.039

Phillips, D., Mitchell, E.J., Lea-Langton,A., Parmar, K. R., Jones,J.,y Williams, A. (2016). The use of conservation biomass feedstocks as potential bioenergy resources in the United Kingdom. Bioresource Technology, 212, 271-279. doi: http://dx.doi.org/10.1016/j.biortech.2016.04.057

Pinedo, A. (2013). Obtención de biocarbonos y biocombustibles mediante pirólisis de biomasa residual (tesis de maestría). Facultad de Ciencias, Departamento de Ciencias Analíticas, Universidad Nacional de Educación a Distancia. Recuperado de http://digital.csic.es/bitstream/10261/80225/1/BIOCARBONES_CENIM_CSIC.pdf

Román-Figueroa, C., Montenegro, N., y Paneque, M. (2016). Bioenergy potential from crop residue biomass in Araucana Region of Chile. Renewable Energy. 102, 170-177 doi: 10.1016/j. renene.2016.10.013

Sagastume, A., Cabello, J., Hens, L, Vandecasteele. (2016). The Biomass Based Electricity Generation Potential of the Province of Cienfuegos. Waste Biomass Valor. doi: DOI 10.1007/ s12649-016-9687-x, pp.1-11

Salazar, I., Mockey, I., y Canal, M. (2009). Estimado de la reducción de la emisión de CO2 por acciones de ahorro de electricidad en las condiciones de Cuba. Ingeniería Energética, 31(3).

Sawin, J. (2016). Energías renovables 2016. Reporte de la situación mundial. París: REN21.

Simangunsong, B., Sitanggang, V., Manurung, E. Rahmadi, A., Moore, G., Aye, L., y Tambunan, A. (2017). Potential forest biomass resource as feedstock for bioenergy and its economic value in Indonesia. Forest Policy and Economics, 81, 10-17.

Stich, S., Hamacher, T., y Stimming, U. (2017). Techno-Economic Estimation of the Power Generation Potential from Biomass Residues in Southeast Asia. Energy, 135, 930-942. doi: 10.1016/j.energy.2017.06.162 
Suzuki, K., Tsuji, N., Shirai, Y., Hassan, M. A., y Osaki, M. (2017). Evaluation of biomass energy potential towards achieving sustainability in biomass energy utilization in Sabah, Malaysia. Biomass and Bioenergy, 97, 149-154. doi: http://dx.doi.org/10.1016/j.biombioe.2016.12.023

Thomas, P., Soren, N., Rumjit, N., James, J., y Saravanakumar, M. (2017). Biomass resources and potential of anaerobic digestion in Indian scenario. Renewable and Sustainable Energy Reviews, 77, 718-730. doi: http://dx.doi.org/10.1016/j.rser.2017.04.053

Toklu, E. (2017). Biomass energy potential and utilization in Turkey. Renewable Energy, 107, 235244. doi: http://dx.doi.org/10.1016/j.renene.2017.02.008

Vávrová, J., Knapek, J., y Weger, J. (2017). Short-term boosting of biomass energy sources - Determination of biomass potential for prevention of regional crisis situations. Renewable and Sustainable Energy Reviews, 67, 426-436. doi: http://dx.doi.org/10.1016/j.rser.2016.09.015

Vavrova, K., Knápek, J., Weger, J., Kralík, T., y Beranovský, J. (2017). Model for evaluation of locally available biomass competitiveness for decentralized space heating in villages and small towns. Renewable Energy, 1-13. doi: http://dx.doi.org/10.1016/j.renene.2017.05.079 
